\title{
TITLE:
}

\section{High-resolution diffraction microscopy using the plane-wave field of a nearly diffraction limited focused $x$-ray beam}

\section{$\operatorname{AUTHOR}(S)$ :}

Takahashi, Yukio; Nishino, Yoshinori; Tsutsumi, Ryosuke; Kubo, Hideto; Furukawa, Hayato; Mimura, Hidekazu; Matsuyama, Satoshi; ... Matsubara, Eiichiro; Ishikawa, Tetsuya; Yamauchi, Kazuto

\section{CITATION:}

Takahashi, Yukio ...[et al]. High-resolution diffraction microscopy using the plane-wave field of a nearly diffraction limited focused $x$-ray beam. PHYSICAL REVIEW B 2009, 80 (5): 054103.

\section{ISSUE DATE:}

2009-08

URL:

http://hdl.handle.net/2433/109871

\section{RIGHT:}

(c) 2009 The American Physical Society 


\title{
High-resolution diffraction microscopy using the plane-wave field of a nearly diffraction limited focused x-ray beam
}

\author{
Yukio Takahashi, ${ }^{1, *}$ Yoshinori Nishino, ${ }^{2}$ Ryosuke Tsutsumi, ${ }^{3}$ Hideto Kubo, ${ }^{3}$ Hayato Furukawa, ${ }^{3}$ Hidekazu Mimura, ${ }^{3}$ \\ Satoshi Matsuyama, ${ }^{3}$ Nobuyuki Zettsu, ${ }^{4}$ Eiichiro Matsubara, ${ }^{5}$ Tetsuya Ishikawa, ${ }^{2}$ and Kazuto Yamauchi ${ }^{3,4}$ \\ ${ }^{1}$ Frontier Research Base for Global Young Researchers, Frontier Research Center, Graduate School of Engineering, Osaka University, \\ 2-1 Yamada-oka, Suita, Osaka 565-0871, Japan \\ ${ }^{2}$ RIKEN SPring-8 Center, Kouto, Sayo, Hyogo 679-5148, Japan \\ ${ }^{3}$ Department of Precision Science and Technology, Graduate School of Engineering, Osaka University, 2-1 Yamada-oka, Suita, \\ Osaka 565-0871, Japan \\ ${ }^{4}$ Research Center for Ultra-Precision Science and Technology, Graduate School of Engineering, Osaka University, 2-1 Yamada-oka, \\ Suita, Osaka 565-0871, Japan \\ ${ }^{5}$ Department of Materials Science and Engineering, Kyoto University, Yoshida, Sakyo, Kyoto 606-8501, Japan
}

(Received 19 May 2009; revised manuscript received 22 June 2009; published 5 August 2009)

\begin{abstract}
X-ray waves in the center of the beam waist of nearly diffraction limited focused $\mathrm{x}$-ray beams can be considered to have amplitude and phase that are both almost uniform, i.e., they are x-ray plane waves. Here we report the results of an experimental demonstration of high-resolution diffraction microscopy using the x-ray plane wave of the synchrotron x-ray beam focused using Kirkpatrik-Baez mirrors. A silver nanocube with an edge length of $\sim 100 \mathrm{~nm}$ is illuminated with the x-ray beam focused to a $\sim 1 \mu \mathrm{m}$ spot at $12 \mathrm{keV}$. A highcontrast symmetric diffraction pattern of the nanocube is observed in the forward far field. An image of the nanocube is successfully reconstructed by an iterative phasing method and its half-period resolution is $3.0 \mathrm{~nm}$. This method does not only dramatically improve the spatial resolution of $\mathrm{x}$-ray microscopy but also is a key technology for realizing single-pulse diffractive imaging using x-ray free-electron lasers.
\end{abstract}

DOI: 10.1103/PhysRevB.80.054103

PACS number(s): 61.05.cf, 07.85.-m, 68.37.Yz

\section{INTRODUCTION}

Microscopy has contributed greatly to our knowledge of the structure and function not only in physics but also various other fields and is an indispensable tool in recent nanoscience and nanotechnology. The recent progress in microscopy techniques is extraordinary. Surface atomic arrangements can be observed by scanning tunneling microscopy or atomic force microscopy, and atomic-scale structures of thin films can be evaluated by transmission electron microscopy (TEM) while x-ray microscopy has lagged behind with respect to resolution compared with the above microscopies because of the difficulty of fabricating $\mathrm{x}$-ray lenses. To avoid this difficulty, a lensless x-ray microscopy combining coherent $\mathrm{x}$-ray scattering and the iterative phasing method, i.e., coherent x-ray diffraction microscopy (CXDM) was proposed by Sayre ${ }^{1}$ in 1980 and demonstrated by Miao et $\mathrm{al}^{2}{ }^{2}$ in 1999. In principle, CXDM provides a spatial resolution close to the $\mathrm{x}$-ray wavelength. Realistic resolution depends on both the quality of $\mathrm{x}$-ray diffraction data over a wide- $q$ region and the uniqueness of phase-retrieval calculations, where $q$ is the magnitude of the scattering vector. Recently, the spatial resolution of CXDM has reached the sub10 -nm scale ${ }^{3,4}$ for two-dimensional images and the sub50 -nm scale for three-dimensional images ${ }^{5,6}$ and is steadily approaching that of electron or probe microscopy. However, it is still not easy to improve the resolution less than $10 \mathrm{~nm}$ since diffraction intensities rapidly decay in the high- $q$ region. In this paper, we propose a method for dramatically improving the spatial resolution of CXDM and report its first demonstration experiment.

Until now, CXDM has been applied to various biological ${ }^{7-10}$ and material ${ }^{11-13}$ imaging tasks because of the interest in the static structure. Promising applications of CXDM in the near future are the single-particle imaging of biomolecules and pump-probe imaging for dynamical structure studies using a single-pulse illumination of $\mathrm{x}$-ray freeelectron lasers (XFELs), which are the next-generation light sources under development in the U.S., Europe, and Japan. These applications have been demonstrated in experiments at a vacuum-ultraviolet free-electron laser facility. ${ }^{14-16}$ In order to realize these challenging applications using XFELs, the XFEL beam must be focused to increase the X-ray photon density to ensure an adequate signal in the high- $q$ region of the diffraction pattern. Focusing devices are necessary to produce a high-density XFEL single pulse. Total-reflection focusing mirrors are considered to be the best device for XFELs because of their high efficiency and radiation hardness. ${ }^{17}$ Therefore, CXDM, in combination with the x-ray beam focused by total-reflection mirrors, is the most promising system for realizing single-pulse XFEL imaging. In this study, high-resolution CXDM with a total-reflection mirrorfocusing device, i.e., Kirkpatrik-Baez (KB) mirrors, ${ }^{18}$ is developed and demonstrated in an experiment at a thirdgeneration synchrotron facility.

\section{CONCEPT OF HIGH-RESOLUTION DIFFRACTION MICROSCOPY USING FOCUSED X-RAY BEAM}

For CXDM using highly focused $\mathrm{x}$-ray beams, ${ }^{4,19}$ it is very important not only to increase the incident coherent $\mathrm{x}$-ray flux but also to decrease the parasitic scattering $\mathrm{x}$ rays, which cause noise from the focusing optics. To meet this demand, KB mirrors were specially designed by wave optical simulation ${ }^{20}$ and fabricated by elastic emission 
(a)

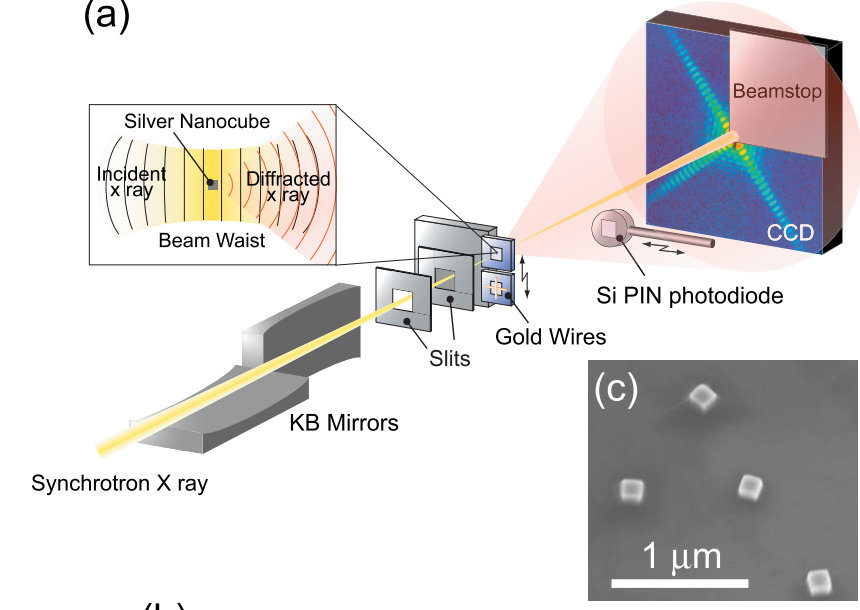

(b)
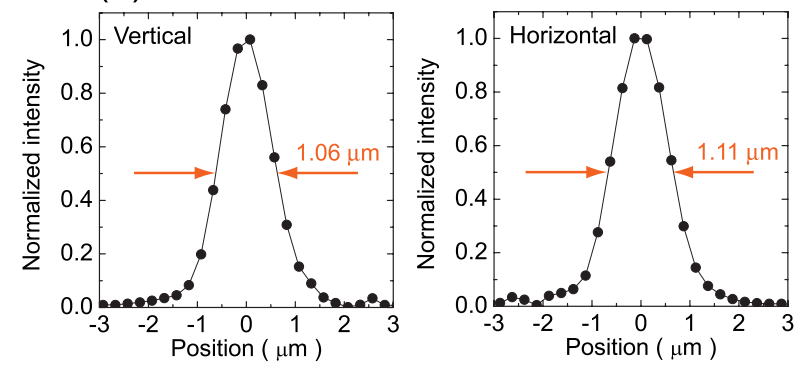

FIG. 1. (Color) (a) Experimental setup for coherent x-ray diffraction pattern measurements of silver nanocube using $\mathrm{x}$ rays focused by KB mirrors. The nanocube was placed at the center of the beam waist. (b) Intensity distribution profile along vertical and horizontal directions of the focused beam. The profile was derived by differentiating the x-ray absorption distribution of the gold wires at $250 \mathrm{~nm}$ intervals. (c) SEM image of silver nanocubes.

machining. ${ }^{21-23}$ The figure error of the fabricated mirrors was less than $1.0 \mathrm{~nm}$ for peak-to-valley error and less than 0.15 $\mathrm{nm}$ for root-mean-square error. The use of these mirrors provided a speckle-free and nearly diffraction limited focused $\mathrm{X}$-ray beam. In the present method, an isolated sample was placed at the center in the waist of the focused x-ray beam. When the sample size is much smaller than the focal spot size, both the amplitude and the phase of the wave field of the $\mathrm{x}$-ray beam illuminated onto the sample are almost uniform, ${ }^{24}$ i.e., $\mathrm{x}$-ray plane wave. Then, if the $\mathrm{x}$-ray absorption by the sample is sufficiently small, a symmetric diffraction pattern is observed in the forward far field. The numerical data symmetrization results in the reduction in photon shot noise. Furthermore, the missing data due to a beamstop are confined within the centrospeckle in this configuration and hence, a part of the centrospeckle can be measured. This is very important for reconstructing a unique image from the diffraction pattern alone. ${ }^{25}$ On the basis of such a concept, a coherent x-ray diffraction pattern of a nanoparticle was measured using the nearly diffraction limited $\mathrm{X}$-ray beam focused by KB mirrors.

\section{EXPERIMENTAL DETAIL}

Figure 1(a) shows a schematic drawing of the CXDM apparatus equipped with a KB mirrors focusing system. The experiment was carried out at BL29XUL in SPring-8. ${ }^{26}$ When the light source is considered to be the electron beam in the vicinity of the undulator exit. The source size, which is defined as the width of the Gaussian distribution, is determined to be $301 \mu \mathrm{m}$ in the vertical direction and $6 \mu \mathrm{m}$ in the horizontal direction when all of the gaps of insertion devices are opened. ${ }^{27}$ The $\mathrm{x}$-ray energy was tuned to $12 \mathrm{keV}$ with the undulator gap and a Si (111) double crystal monochromator. The monochromatic $\mathrm{x}$ rays passed through the first cross slit, which was placed at $\sim 50$ m downstream of the source, of $100 \mu \mathrm{m}$ opening for both directions. The KB mirrors were placed at $\sim 100 \mathrm{~m}$ downstream of the source. The second cross slit was placed immediately before the KB mirrors to control the X-ray illumination area to the mirrors. The size of the second slit was adjusted to $100 \mu \mathrm{m}$ for both directions. The x-ray beam was two dimensionally focused at $445 \mathrm{~mm}$ downstream of the second mirror by the KB mirrors that were controlled by a specially designed manipulation system. ${ }^{28}$ The higher-order harmonics of the undulator radiation were cut down to $1 \%$ by double reflections. Intensity of the reflected $\mathrm{x}$ rays was $\sim 2 \times 10^{11}$ photons/s which was almost equal to that immediately after the second slit. The $\mathrm{X}$-ray reflectivity was close to $100 \%$. The sample or the gold wire of $200 \mu \mathrm{m}$ in the diameter can be inserted in the focus. When the focal profile was measured by the wire scanning method, a Si-PIN photodiode was inserted after the wire. Figure 1(b) shows the measured focal profiles for vertical and horizontal directions. The full width at half maximum of the focal profiles was $\sim 1 \mu \mathrm{m}$ along both directions. In order to interrupt scattering $\mathrm{x}$ rays from the mirrors, two guard slits were placed between the mirrors and the focal point.

Silver nanocubes with an edge length of $\sim 100 \mathrm{~nm}$ were prepared as samples. The amount of X-ray absorption of the single nanocube was less than $1 \%$. Figure $1(\mathrm{c})$ shows a scanning electron microscope (SEM) image of the nanocubes. The nanocubes were single crystals characterized by a slightly truncated shape bounded by $\{100\},\{110\}$, and $\{111\}$ facets. ${ }^{29}$ The nanocubes were mounted on a 100 -nm-thick $\mathrm{SiN}$ membrane. In order to prevent $\mathrm{x}$-ray-induced charge up, a carbon film a few nm thick was deposited beforehand on the $\mathrm{SiN}$ membrane chip. An isolated nanocube was selected and illuminated with the focused x-ray beam. The forward diffracted x-ray photons were detected by an in-vacuum front-illuminated charge-coupled device (CCD) detector with a pixel size of $20 \times 20 \mu^{2}$ placed at $999 \mathrm{~mm}$ downstream of the sample. A beamstop was placed in front of the CCD detector. In order to cover a wide dynamic range, the lowand high- $q$ diffraction data were separately collected by changing the beamstop position. The exposure time of $\mathrm{x}$ rays for the low- and high- $q$ diffraction data was 100 and $800 \mathrm{~s}$, respectively.

\section{RESULTS AND DISCUSSION}

\section{A. Diffraction pattern}

Figure 2(a) shows the measured coherent diffraction pattern of a silver nanocube, where low- and high- $q$ data are combined and symmetrized. Interference fringes with a high 


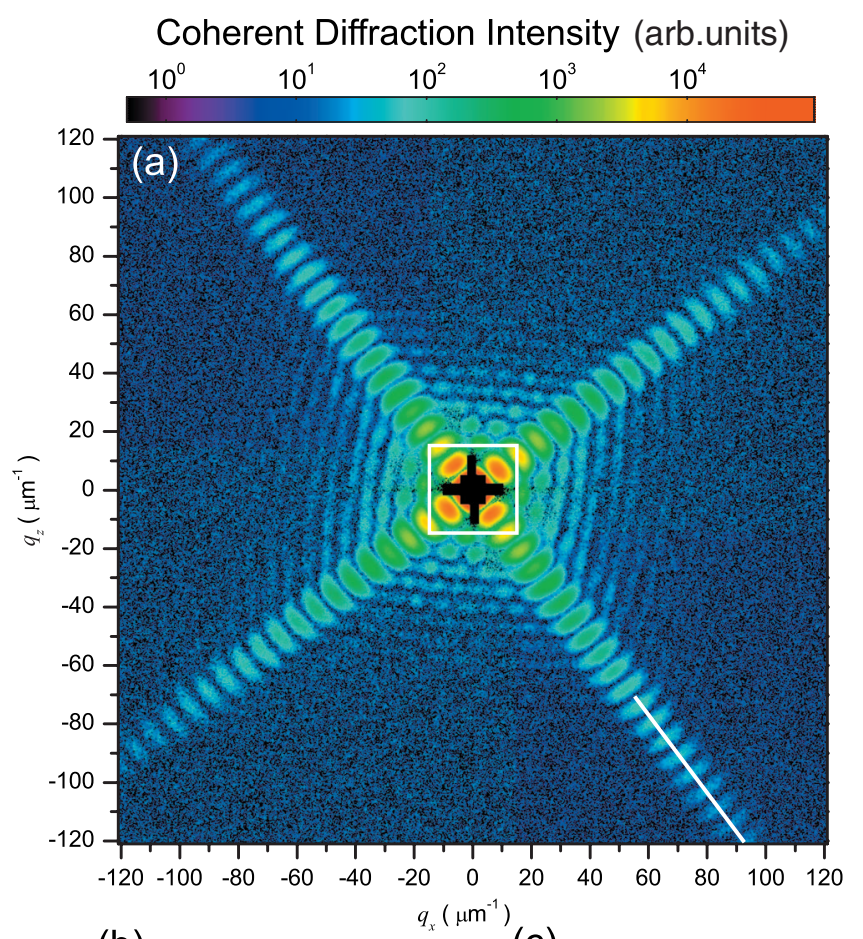

(b)
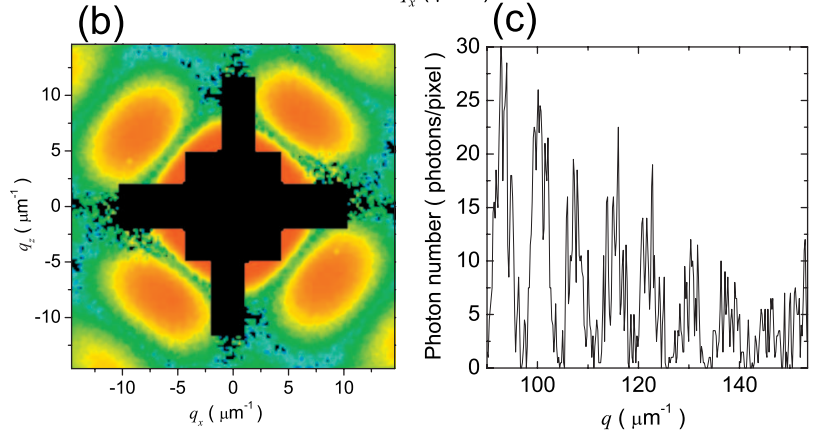

FIG. 2. (Color) (a) Coherent diffraction pattern of silver nanocube in $1251 \times 1251$ pixels. $\boldsymbol{q}$ is defined as $|\boldsymbol{q}|$ $=2 \sin (\Theta / 2) / \lambda$, where $\Theta$ is the scattering angle and $\lambda$ is the $x$-ray wavelength. (b) Expanded image of the area inside the square in (a). (c) $q$ dependence, along the white line indicated in (a), of photon numbers detected at one pixel of the CCD detector for the high- $q$ diffraction measurement.

visibility are observed over a wide- $q$ range, which means that the degree of coherence of the focused beam was high and the nanocube was appropriately set up in the vicinity of the focus. ${ }^{24}$ Clear speckle patterns extending crosswise reflects the cubic shape of the particle. The central black region is an unmeasured region owing to the beamstop. The black region extending vertically and horizontally from the center is an unmeasurable region owing to the scattering of $\mathrm{x}$ rays by the mirrors. Figure 2(b) shows the enlarged image of the low- $q$ region surrounding the white square in Fig. 2(a). A part of the red-colored central speckle can be seen, which is important in reconstructing a unique image by the iterative phasing method. ${ }^{25}$ Figure 2(c) shows the $q$ dependence of photon numbers detected in the high- $q$ region along the white line indicated in Fig. 2(a). Approximately five photons were detected at $145 \mu \mathrm{m}^{-1}$, which means that structures with $3 \mathrm{~nm}$ were resolved by the $\mathrm{x}$-ray diffraction. (a)

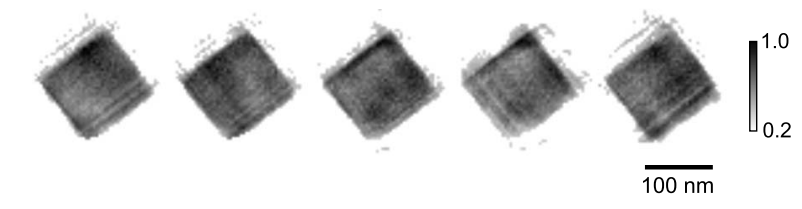

(b)

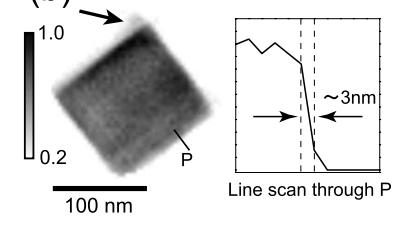

(c)

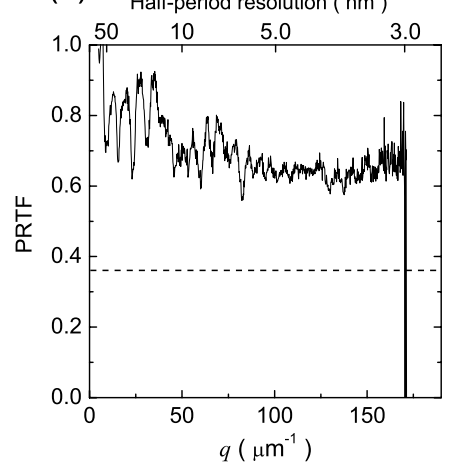

FIG. 3. (a) Five projection images of the silver nanocube reconstructed from the diffraction pattern in Fig. 2(a), which were started from different electron-density maps. (b) Final image derived by averaging the five images of (a) in which a line scan through an edge shown in the inset demonstrates the resolution of $\sim 3 \mathrm{~nm}$. The reconstructed images are normalized by the maximum value of image intensities and are displayed in gray scale. (c) PRTF for the reconstructed image of (b). The half-period resolution of the image of (b) is $3.0 \mathrm{~nm}$, where the PRTF drops to a value of 1/e. (d) SEM image of the silver nanocube.

\section{B. Reconstructed image}

The image of the silver nanocube was reconstructed by the hybrid input-output method ${ }^{30,31}$ with Shrink-Wrap algorithm. ${ }^{32}$ The iterative process started from a random electron-density map in a square support and the support was revised iteratively every 1000 iterations using immediately reconstructed images. Here, the support is a nonzero region in real space. In the iterative process, the electron density outside the support is forced to decrease gradually in real space and the measured coherent diffraction data is used in reciprocal space. The iterative process was continued for up to $2 \times 10^{3}$ iterations. Two-dimensional images of the silver nanocube were reconstructed starting from ten different random electron-density maps and the five most similar resultant images were averaged to obtain the final reconstruction.

Figure 3(a) shows the selected five reconstructions which are displayed in gray scale. Although the square shape of the nanocube is clearly reconstructed, the intensity distributions of five image intensities are not unique. This is due to both the lack of a part of the diffraction data around the centrospeckle and photon shot noise. Figure 3(b) shows the averaged image for the final reconstruction. The spatial resolution of the final reconstruction was estimated by computing the phase-retrieval transfer function (PRTF) (Refs. 5 and 8) shown in Fig. 3(c). The value of the resolution was defined by the position where the PRTF drops to $1 / e .{ }^{8,33}$ According to this manner, the present image achieves a $3.0 \mathrm{~nm}$ halfperiod resolution. It is understood that the nonunique distri- 
bution of the images intensity causes to be PRTF $<1$ and the 3.0-nm resolution results from the sharp edge structure of the cubic side due to $\{100\}$ facet.

Figure 3(d) shows the SEM image of the identical nanocube before irradiating $x$ rays. SEM images reflect the surface structure while the CXDM image reflects the electron-density distribution of the sample. Image intensities of Fig. 3(b) are proportional to the projection of the electron density in the direction of the incident x-ray beam. A small convex structure was observed in both images, which is indicated by an arrow. In the CXDM image, the image intensity of the small convex structure is small. It is guessed that this convex structure is thin and/or includes low-electrondensity regions.

\section{Possibility of single-pulse diffraction microscopy using focused XFEL beams}

From the viewpoint of the number of $\mathrm{x}$-ray photons, the possibility of single-pulse CXDM using focused XFEL beams is discussed. The number of X-ray photons illuminated onto the silver nanocube can be estimated from the product of the $\mathrm{x}$-ray photon flux and the irradiation area of the silver nanocube. The $\mathrm{x}$-ray photon flux per second within $100 \times 100 \mathrm{~nm}^{2}$ around the focal point was $\sim 1.0$ $\times 10^{4}$ photons $/ \mathrm{nm}^{2}$, which was estimated using both the shape of the focal profiles and the X-ray intensities measured with the Si-PIN photodiode. From the reconstructed images in Fig. 3(b), the irradiation area of the nanocube was found to be $1.68 \times 10^{4} \mathrm{~nm}^{2}$. The number of $\mathrm{x}$-ray photons illuminated onto the silver nanocube in low- and high- $q$ measurements was $\sim 1.5 \times 10^{11}$ photons. The peak photon flux obtained at the Japanese XFEL facility is estimated to be $\sim 2$ $\times 10^{11}$ photons/pulse. ${ }^{34}$ If the single-pulse photons are suitably focused and illuminated onto the same nanocube, equivalent diffraction data to that of the present experiment should be collected.

\section{CONCLUSION}

In the near future, various nanoparticles and biological samples will be two or three dimensionally observed by the present technique. Similar observations can be performed also by the well-established TEM techniques, such as high- resolution TEM and TEM tomography. Compared with TEM, CXDM is excellent in the point of the quantitative evaluation of the electron-density distribution. ${ }^{35}$ In addition, element-specific CXDM (Refs. 36-38) using the X-ray absorption edge will be easily realized since the focal position changes negligibly even if incident $\mathrm{X}$-ray energies are changed. By complementarily using CXDM and TEM, it will become possible to understand the nanostructure directly linked with those properties in detail. When the samples are illuminated with high-flux $\mathrm{x}$ rays, note that the radiation damage to the samples, particularly biological samples, becomes serious. Cryogenetic systems will be required to alleviate the radiation damage problem. ${ }^{39}$ The present mirrorfocusing system can be applied to keyhole coherent diffractive imaging ${ }^{40}$ and ptychographic imaging ${ }^{41}$ which make possible the imaging of extended objects. In these methods, it is necessary to exactly determine the x-ray illumination function. Theoretical understanding and experimental determination of the wave field of $\mathrm{x}$-ray beams focused by $\mathrm{KB}$ mirrors will be required, as has been carried out for a Fresnel zone plate. ${ }^{42}$ We believe that CXDM in combination with KB mirror-focusing opens up a new frontier of $\mathrm{x}$-ray microscopy science using synchrotron $\mathrm{x}$ rays and will become a key technology in realizing single-pulse CXDM using XFELs in the near future.

\section{ACKNOWLEDGMENTS}

This research has been carried out at the Frontier Research Base for Global Young Researchers, Osaka University, in the Program of Promotion of Environmental Improvement to Enhance Young Researcher's Independence, the Special Coordination Funds for Promoting Science and Technology from the Ministry of Education, Culture, Sports, Science and Technology (MEXT). This work was also partly supported by funds from a Grant-in-Aid for the "Promotion of X-ray Free Electron Laser Research," Specially Promoted Research (Grant No. 18002009), Young Scientists (Grant No. 21686060), the Global COE Program "Center of Excellence for Atomically Controlled Fabrication Technology" from MEXT, Konica Minolta Imaging Science Foundation, Iketani Science and Technology Foundation, and Shimadzu Science Foundation. The authors would like to acknowledge $\mathrm{H}$. Yumoto for providing experimental advice.

\footnotetext{
*Corresponding author; takahashi@wakate.frc.eng.osaka-u.ac.jp

${ }^{1}$ D. Sayre, in Imaging Processes and Coherence in Physics, Springer Lecture Notes in Physics Vol. 112, edited by J. Schlenker, M. Fink, J. P. Goedgebuer, V. Malgrange, J. C. Viénot, and R. H. Wade (Springer, Berlin, 1980), pp. 229-235.

${ }^{2}$ J. Miao, P. Charalambous, J. Kirz, and D. Sayre, Nature (London) 400, 342 (1999).

${ }^{3}$ J. Miao, T. Ishikawa, E. H. Anderson, and K. O. Hodgson, Phys. Rev. B 67, 174104 (2003).

${ }^{4}$ C. G. Schroer, P. Boye, J. M. Feldkamp, J. Patommel, A. Schropp, A. Schwab, S. Stephan, M. Burghammer, S. Schoder,
}

and C. Riekel, Phys. Rev. Lett. 101, 090801 (2008).

${ }^{5}$ H. N. Chapman, A. Barty, S. Marchesini, A. Noy, S. P. HauRiege, C. Cui, M. R. Howells, R. Rosen, H. He, J. C. H. Spence, U. Weierstall, T. Beetz, C. Jacobsen, and D. Shapiro, J. Opt. Soc. Am. A 23, 1179 (2006).

${ }^{6}$ O. M. Yefanov, A. V. Zozulya, I. A. Vartanyants, J. Stangl, C. Mocuta, T. H. Metzger, G. Bauer, T. Boeck, and M. Schmidbauer, Appl. Phys. Lett. 94, 123104 (2009).

${ }^{7}$ J. Miao, K. O. Hodgson, T. Ishikawa, C. A. Larabell, M. A. LeGros, and Y. Nishino, Proc. Natl. Acad. Sci. U.S.A. 100, 110 (2003). 
${ }^{8}$ D. Shapiro, P. Thibault, T. Beetz, V. Elser, M. Howells, C. Jacobsen, J. Kirz, E. Lima, H. Miao, A. M. Neiman, and D. Sayre, Proc. Natl. Acad. Sci. U.S.A. 102, 15343 (2005).

${ }^{9}$ C. Song, H. Jiang, A. Mancuso, B. Amirbekian, L. Peng, R. Sun, S. S. Shah, Z. H. Zhou, T. Ishikawa, and J. Miao, Phys. Rev. Lett. 101, 158101 (2008).

${ }^{10}$ Y. Nishino, Y. Takahashi, N. Imamoto, T. Ishikawa, and K. Maeshima, Phys. Rev. Lett. 102, 018101 (2009).

${ }^{11}$ M. A. Pfeifer, G. J. Williams, I. A. Vartanyants, R. Harder, and I. K. Robinson, Nature (London) 442, 63 (2006).

${ }^{12}$ Y. Takahashi, Y. Nishino, T. Ishikawa, and E. Matsubara, Appl. Phys. Lett. 90, 184105 (2007).

${ }^{13}$ A. Barty, S. Marchesini, H. N. Chapman, C. Cui, M. R. Howells, D. A. Shapiro, A. M. Minor, J. C. H. Spence, U. Weierstall, J. Ilavsky, A. Noy, S. P. Hau-Riege, A. B. Artyukhin, T. Baumann, T. Willey, J. Stolken, T. van Buuren, and J. H. Kinney, Phys. Rev. Lett. 101, 055501 (2008).

${ }^{14}$ K. J. Gaffney and H. N. Chapman, Science 316, 1444 (2007).

${ }^{15}$ M. J. Bogan, W. H. Benner, S. Boutet, U. Rohner, M. Frank, A. Barty, M. M. Seibert, F. Maia, S. Marchesini, S. Bajt, B. Woods, V. Riot, S. P. Hau-Riege, M. Svenda, E. Marklund, E. Spiller, J. Hajdu, and H. N. Chapman, Nano Lett. 8, 310 (2008).

${ }^{16}$ A. Barty, S. Boutet, M. J. Bogan, S. Hau-Riege, S. Marchesini, K. Sokolowski-Tinten, N. Stojanovic, R. Tobey, H. Ehrke, A. Cavalleri, S. Düsterer, M. Frank, S. Bajt, B. W. Woods, M. M. Seibert, J. Hajdu, R. Treusch, and H. N. Chapman, Nat. Photonics 2, 415 (2008).

${ }^{17}$ H. Mimura, S. Morita, T. Kimura, D. Yamakawa, W. Lin, Y. Uehara, S. Matsuyama, H. Yumoto, H. Ohashi, K. Tamasaku, Y. Nishino, M. Yabashi, T. Ishikawa, H. Ohmori, and K. Yamauchi, Rev. Sci. Instrum. 79, 083104 (2008).

${ }^{18}$ P. Kirkpatrick and A. V. Baez, J. Opt. Soc. Am. A Opt. Image Sci. Vis 38, 766 (1948).

${ }^{19}$ G. J. Williams, H. M. Quiney, B. B. Dhal, C. Q. Tran, K. A. Nugent, A. G. Peele, D. Paterson, and M. D. de Jonge, Phys. Rev. Lett. 97, 025506 (2006).

${ }^{20}$ Y. Takahashi, Y. Nishino, H. Mimura, R. Tsutsumi, H. Kubo, T. Ishikawa, and K. Yamauchi, J. Appl. Phys. 105, 083106 (2009).

${ }^{21}$ Y. Mori, K. Yamauchi, and K. Endo, Precis. Eng. 9, 123 (1987).

${ }^{22}$ Y. Mori, K. Yamauchi, and K. Endo, Precis. Eng. 10, 24 (1988).

${ }^{23}$ K. Yamauchi, H. Mimura, K. Inagaki, and Y. Mori, Rev. Sci. Instrum. 73, 4028 (2002).

${ }^{24}$ I. Robinson, F. Pfeiffer, I. Vartanyants, Y. Sun, and Y. Xia, Opt. Express 11, 2329 (2003).

${ }^{25}$ J. Miao, Y. Nishino, Y. Kohmura, B. Johnson, C. Song, S. H. Risbud, and T. Ishikawa, Phys. Rev. Lett. 95, 085503 (2005).

${ }^{26}$ K. Tamasaku, Y. Tanaka, M. Yabashi, H. Yamazaki, N. Kawa- mura, M. Suzuki, and T. Ishikawa, Nucl. Instrum. Methods Phys. Res. A 467-468, 686 (2001).

${ }^{27}$ See accelerator overview in SPring- 8 website http:// www.spring8.or.jp

${ }^{28}$ S. Matsuyama, H. Mimura, H. Yumoto, H. Hara, K. Yamamura, Y. Sano, K. Endo, Y. Mori, M. Yabashi, Y. Nishino, K. Tamasaku, T. Ishikawa, and K. Yamauchi, Rev. Sci. Instrum. 77, 093107 (2006).

${ }^{29}$ Y. Sun and Y. Xia, Science 298, 2176 (2002).

${ }^{30}$ J. R. Fienup, Appl. Opt. 21, 2758 (1982).

${ }^{31}$ Y. Nishino, J. Miao, and T. Ishikawa, Phys. Rev. B 68, 220101(R) (2003).

${ }^{32}$ S. Marchesini, H. He, H. N. Chapman, S. P. Hau-Riege, A. Noy, M. R. Howells, U. Weierstall, and J. C. H. Spence, Phys. Rev. B 68, 140101(R) (2003).

${ }^{33}$ H. N. Chapman, A. Barty, M. J. Bogan, S. Boutet, M. Frank, S. P. Hau-Riege, S. Marchesini, B. W. Woods, S. Bajt, W. H. Benner, R. A. London, E. Plönjes, M. Kuhlmann, R. Treusch, S. Düsterer, T. Tschentscher, J. R. Schneider, E. Spiller, T. Möller, C. Bostedt, M. Hoener, D. A. Shapiro, K. O. Hodgson, D. V. D. Spoel, F. Burmeister, M. Bergh, C. Chaleman, G. Huldt, M. M. Seibert, F. R. N. C. Maia, R. W. Lee, A. Szöke, N. Timneanu, and J. Hajdu, Nat. Phys. 2, 839 (2006).

${ }^{34}$ T. Shintake, Proceedings of the 11th European Particle Accelerator Conference (EPAC'08), 2008, http://epaper.kek.ip/e08/ index.htm, p. 136.

${ }^{35}$ J. Miao, J. Amonette, Y. Nishino, T. Ishikawa, and K. O. Hodgson, Phys. Rev. B 68, 012201 (2003).

${ }^{36}$ C. Song, R. Bergstrom, D. Ramunno-Johnson, H. Jiang, D. Paterson, M. D. de Jonge, I. McNulty, J. Lee, K. L. Wang, and J. Miao, Phys. Rev. Lett. 100, 025504 (2008).

${ }^{37}$ Y. Takahashi, H. Kubo, H. Furukawa, K. Yamauchi, E. Matsubara, T. Ishikawa, and Y. Nishino, Phys. Rev. B 78, 092105 (2008).

${ }^{38}$ A. Scherz, D. Zhu, R. Rick, W. F. Schlotter, S. Roy, J. Lüning, and J. Stöhr, Phys. Rev. Lett. 101, 076101 (2008).

${ }^{39}$ T. Beetz, M. R. Howells, C. Jacobsen, C.-C. Kao, J. Kirz, E. Limaa, T. O. Mentes, H. Miao, C. Sanchez-Hanke, D. Sayre, and D. Shapiro, Nucl. Instrum. Methods Phys. Res. A 545, 459 (2005).

${ }^{40}$ B. Abbey, K. A. Nugent, G. J. Williams, J. N. Clark, A. G. Peele, M. A. Pfeifer, M. de Jonge, and I. McNulty, Nat. Phys. 4, 394 (2008).

${ }^{41}$ P. Thibault, M. Dierolf, A. Menzel, O. Bunk, C. David, and F. Pfeiffer, Science 321, 379 (2008).

${ }^{42}$ H. M. Quiney, A. G. Peele, Z. Cai, D. Paterson, and K. A. Nugent, Nat. Phys. 2, 101 (2006). 